\title{
Attitude Survey of Youth for Mutual Aid Support System for Local Community Using Bayesian Network
}

\author{
Shimpei Matsumoto *, Nobuyuki Ohigashi *
}

\begin{abstract}
Some Hiroshima citizens live in suburban residential estates at the slope of mountains surrounding the city, but the living environment is unsuitable for "vulnerable road users" mainly elderly people. To improve their living environments, we have shown the concept of information sharing system to support them living in regions where public transport is not sufficiently developed. In addition, we have constructed a web service to support their daily life named MASS. Our system's role is to facilitate the encounter between local community people, provide the opportunity of resource sharing, generate some kinds of transport means, and solve the difficulties in daily life by mutual aid. Young people's active participation is essential to make our system more effective for vulnerable road users because most of the skills will be provided by young people. The analysis of the previous work unveiled a trend of the youth consciousness about skill sharing in their local community and found the limitation of Quantification Method Type II. Therefore, in this paper, to discuss our system's continuity as a business, we conducted an attitude survey on young people's awareness of resource sharing in their local community and analyzed it with Bayesian network. From the experimental result, we re-confirmed the findings of the previous research and further clarified the relationship between the factors that we could not clarify so far. In addition, we conducted numerical experiments using the constructed Bayesian network, obtained results that support several hypotheses, and revealed factors that have a strong influence on the use of MASS.
\end{abstract}

Keywords: Vulnerable road users, resource-sharing, mutual aid, local community activation, Bayesian network.

\section{Introduction}

Most of the residential estates in the surrounding mountains of Hiroshima City are developed under the theory of neighborhood unit [1]. Still, they are located on sloping ground and are far from the central area. So, these living environments would be unsuitable for "vulnerable road users" mainly elderly people. Based on the background, we have examined a new public transport means to keep the residential service level of these areas in good

\footnotetext{
* Hiroshima Institute of Technology, Hiroshima, Japan
} 
condition for the longest possible time. In our previous studies, we had shown the concept of a transport support model for "vulnerable road users" mainly elderly people living in the suburban slope residential areas whose public transports are insufficient. We have also designed and developed a prototype of Web service [2][3] called MASS (Mutual Aid Support System). Today, resource sharing services like goodtiming ${ }^{1}$, which encourage local residents to help each other, frequently appear in recent years. But at that time, the concept of MASS was ahead of skill-sharing services [4][5] for solving regional problems by regional resources. MASS can provide efficient transport means for vulnerable road users and municipalities by utilizing local residents' help, which is just the concept of sharing economy. In addition, to further support the vulnerable road user's daily life, we had improved this prototype to a skill-sharing service [6]-[9]. With MASS, for example, a resident, who will move to his/her destination with one's mobility, such as a private car, can help another resident's trouble (mainly older people) at the same time as his/her original purpose. We expect that the mutual aid generated by MASS will enhance local people's relationship building and the regeneration of the local community.

Young people's positive participation would be indispensable for the activation of MASS because most of the services will be from young people. Many of the sharing services have been successful, thanks to the active use of young people [10]-[13]. Therefore, in our previous studies, in order to discuss the continuity of MASS as a business, we have conducted an attitude survey on young people's awareness of resource sharing in their local community. In our previous study [9], we conducted an attitude survey on young people's awareness of resource sharing in their local community and analyzed it with Quantification Method Type II. The analysis result unveiled a trend of the youth consciousness about skill sharing in their local community and found the limitation of Quantification Method Type II. In addition, the previous study has not reached the verification of the hypothesis and the acquisition of knowledge that determine the evaluation of MASS because the mechanism of the impression for MASS is complicated. We assume that there is a structure where factors as explanatory variables have relationships with each other. Therefore, in this paper, we consider Bayesian network [14]-[18] an effective method to clarify the relationship between explanatory variables and comprehend the mechanism of young people's judgment for MASS. Concretely, this paper shows the analysis results of young people's awareness of MASS with Bayesian network. From the experimental result, we could re-confirm the findings of the previous research and further clarify the relationship between the factors that we have not been able to confirm so far. The experimental results suggested that although young people do not need MASS to interact with and contribute to the region, they may have accepted MASS as a general CtoC service. In addition, we conducted numerical experiments using the constructed Bayesian network, obtained results that support several hypotheses, and revealed factors that have a strong influence on the use of MASS. Especially, we found a possibility that the psychological resistance to engaging with others has a strong influence on MASS. Note that the contribution of this paper is to consider the previously reported findings [19] in more detail.

\section{Mutual Aid Support System}

The core service of MASS is to enhance the encounter between local community people [6][9]. MASS facilitates the encounter by personal information sharing, such as each one's

\footnotetext{
${ }^{1}$ http://goodtiming.jp/
} 
skills and troubles, and aims to solve a troubled resident's problem in daily life. MASS provides the primary two services: the time-dependent skill/trouble information sharing to coordinate someone's request and acceptance, and the other is the communication support to negotiate the condition of mutual aid. Most troubles might be on a daily short trip, but the requests are not limited to these and include gardening, dog-walking, and household appliances maintenance. With MASS, a troubled person, who has no daily transportation means but wants to go out, can find a person who lives near the troubled person and ask to bring out together. Although such mutual aid is a volunteer activity, a helper can obtain decent wages as a donation, and MASS securely manages all transactions of the charges between users.

The flow of resource sharing is as follows:

1. A troubled resident called "client" posts a request to MASS. For example, a resident, who wants to go out but does not have a transportation means or needs someone's help, sends a request.

2. Suppose a user called a "server" who readies to help another decides to accept the request after checking the details of another resident's request, such as conditions and personal information. In that case, MASS connects the server and the client by the direct message function. These users can negotiate the communication function and aim to reach an agreement. MASS will promote the rationalization and the efficiency of transports on people and goods.

3. Since MASS encourages each resident's meetings, the authors expect that MASS can contribute to reproducing a local community.

\section{Bayesian Network}

Constructing a structured graph while implicitly assuming a causal structure is considered effective in narrowing down the combination and reducing the analysis time. In fact, it is obvious from the fact that data scientists generally do something similar. Bayesian network is one of the ways to realize such a procedure mathematically. It can trace and analyze causal relationships existing among many items.

Bayesian network is a model that approximates the simultaneous distribution of discrete probability distributions by an acyclic directed graph network structure with random variables as nodes and conditional probability parameter sets. Especially it focuses on the causal relationship between explanatory variables [20][21], and can represent the dependencies graphically. Sometimes data scientists use Bayesian networks to model relationships between each event from multivariate data, and the model is available to predict and infer. The constructed model of Bayesian network can clarify the relationship between objective variables and explanatory variables and the relationship between explanatory variables. Since we can apply Bayesian network to various data of nonlinear relationships, the range of applications is wide.

In general questionnaires, a causal relationship surely exists between the attributes, like that the final evaluation result depends on the basic attributes and psychological reasons of respondents. For example, if the respondent is introversive, his/her motivation for interacting with others will decrease. In the case of MASS, the mechanism of the reason for deciding its evaluation is complicated. So, we assume that there is a relationship between 
factors as explanatory variables. In other words, the value of the objective variable is obtained after many factors have influenced each other. This structure can be thought of as a complex network. Regression analysis, which is generally used, can reveal the relationship between objective variables and explanatory variables but cannot clarify the relationship between explanatory variables. Bayesian network can show the relationship between the objective variable and the explanatory variable and the relationship between the factors as explanatory variables at the same time. Bayesian network can describe various factors related to the impression on MASS as one network. Thus, we expect that Bayesian network can clarify the structure of the factors leading to the final evaluation. The mechanism of the impression on the regional resource sharing can be better described. From the above reason, we determine Bayesian network effective for clarifying young people's thinking process to MASS. In this paper, we apply Bayesian network to the questionnaire result, which investigated the consciousness of the sharing system to support the vulnerable road users and analyze what kind of characteristics customers had, what they expected, and how they evaluated MASS.

As mentioned above, Bayesian network is a graphical model expressing the dependencies between nodes by conditional probability. Each node is connected by a directed link and the link from node $X$ to node $Y$ means that $Y$ is directly affected by $X$. That is, it means that there is a causal relationship between the directions of the links. The directed link of Bayesian network represents the dependency relation between the random variables, and the dependency can be expressed quantitatively by the conditional probability table. A conditional probability table based on cross-tabulation is created between nodes linked and calculated as a prior probability. The designer's prior knowledge is applicable to network structure and conditional probability tables. The probability value of the objective variable can be estimated by setting the already obtained data to a node of the learned network as evidence. When we confirm that some parameters have occurred (evidence), a posteriori probability is calculated by giving the evidence. For example, if the fact that the parameter $y_{1}$ on node $Y$ is observed, the evidence is set to $y_{1}=1.0$. At this time, the posterior probability of the parameter in the node $X$ can be obtained by calculating $P\left(x_{1} \mid y_{1}\right)$ and $P\left(x_{2} \mid y_{1}\right)$. In this way, probability calculation by evidence setting is called stochastic inference. It can be used for decision-making by changing the probability value of an event by sensitivity analysis.

\section{Experimental Result}

\subsection{Condition of Analysis}

We conduct an attitude survey on young people's awareness for sharing people's resources in the local community. We employed 88 Hiroshima residents from 20 years old to 24 years old as examinees and obtained the questionnaire's awareness data. Each response of the questionnaire was on the 4-grade Likert scale (strongly agree, weakly agree, weakly disagree, strongly disagree); 4 is the maximum (positive), and 1 is the minimum (negative). In this paper, we binarized the responses and analyzed them by Bayesian network, i.e., positive evaluations ( 3 and 4 ) were transformed into 1, otherwise 0 . First, examinees listened to about 10 minutes of presentation on MASS. Then they operated the prototype of MASS freely with sufficient time while receiving its explanation to operate from an experimenter. After that, each examinee answered his/her opinion for each item in the questionnaire. First of all, we confirmed whether the examinees sufficiently understood the concept of MASS 
to check the reliability of the responses. As a result, we confirmed that all reactions are reliable to analyze. First, we asked examinees for their subjective opinion on MASS by following two questions:

\section{Evaluation of MASS}

- $\mathrm{Q}_{1}$ : When MASS is actually launched, would you like to use it as a user to provide your resource?

- $\mathrm{Q}_{2}$ : When MASS is actually launched, would you like to use it as a user to get another one's resource?

$\mathrm{Q}_{1}$ and $\mathrm{Q}_{2}$ are evaluations as to whether the examinees actually wanted to use MASS.

We examined the reasons for the response of $\mathrm{Q}_{1}$ by multiple selection methods. Positive reasons (abbreviated as $\mathrm{P}_{1}-\mathrm{P}_{4}$ ) and negative reasons (abbreviated as $\mathrm{N}_{1}-\mathrm{N}_{4}$ ) are as follows.

\section{$\underline{\text { Positive reasons }}$}

- $\mathrm{P}_{1}$ : I am interested in a new service.

- $\mathrm{P}_{2}$ : I want to get rewards.

- $\mathrm{P}_{3}$ : I want to make a new connection with other people in the local community.

- $\mathrm{P}_{4}$ : I want to contribute to the local community.

$\underline{\text { Negative reasons }}$

- $\mathrm{N}_{1}$ : I am nervous (scared) to engage with unknown others.

- $\mathrm{N}_{2}$ : I do not want to disclose personal information.

- $\mathrm{N}_{3}$ : I do not go out much.

- $\mathrm{N}_{4}$ : I am not interested in making money in such this way.

Similarly, we got the reasons for the response of $\mathrm{Q}_{2}$ by multiple selection methods. Positive reasons (abbreviated as $\mathrm{P}_{5}-\mathrm{P}_{7}$ ) and negative reasons (abbreviated as $\mathrm{N}_{5}-\mathrm{N}_{8}$ ) are as follows.

\section{$\underline{\text { Positive reasons }}$}

- $\mathrm{P}_{5}$ : I want to join the interaction with local people.

- $\mathrm{P}_{6}$ : I want to reduce waste/to save money.

- $\mathrm{P}_{7}$ : I feel MASS useful.

Negative reasons

- $\mathrm{N}_{5}$ : I am not interested in the local community.

- $\mathrm{N}_{6}$ : I feel MASS is useful, but it is uneasy to interact with unknown people.

- $\mathrm{N}_{7}$ : I do not need another's help because I can do my own thing myself.

- $\mathrm{N}_{8}$ : I am worried about some kinds of accidents/troubles.

We constructed a Bayesian network with $\mathrm{Q}_{1}$ and $\mathrm{Q}_{2}$ as objective variables respectively and $\mathrm{P}_{1}$ to $\mathrm{P}_{7}, \mathrm{~N}_{1}$ to $\mathrm{N}_{8}$ as explanatory variables. That is, all of the positive and negative reasons are nodes, and $\mathrm{Q}_{1}$ and $\mathrm{Q}_{2}$ are nodes having no children. In this study, we inputted the questionnaire result as learning data and constructed the model automatically using the information reference amount by AIC as the threshold value. Greedy Search built the model. 


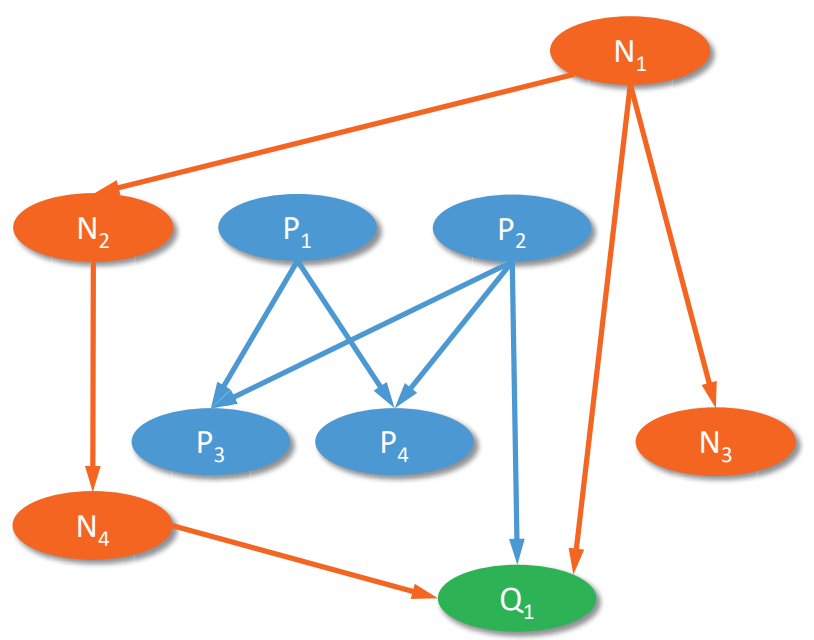

Figure 1: Bayesian network with objective variable $\mathrm{Q}_{1}$

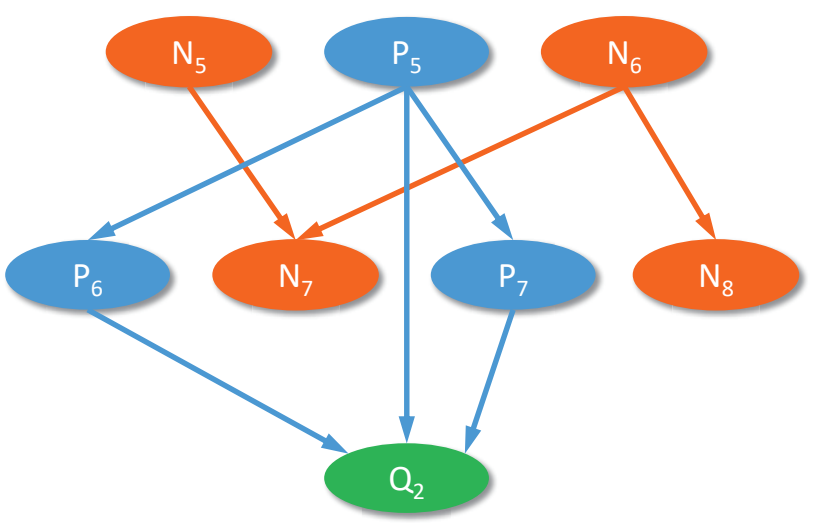

Figure 2: Bayesian network with objective variable $\mathrm{Q}_{2}$

We terminated the search when the average value of cross-tabulation became the threshold value of 0.01 or less. There would be a causal relationship among the same type of reasons, but causalities between different types of reasons like the relationship between positivenegative reasons are unrealistic. Therefore, when constructing the model, we restricted the causal relationship between positive and negative reasons.

\subsection{Analysis Result}

Fig. 1 and Fig. 2 shows Bayesian networks constructed automatically. The prediction accuracy of the constructed model was $100 \%$ for both Q1 and Q2 models. Fig. 1 shows that the three factors are directly related to the evaluation of $\mathrm{Q}_{1}$.In this figure, "contribution to the local community" and "interaction with it" are only indirect factors, so it is inferred that young people expect compensation more than regional contributions. $\mathrm{N}_{1}$ is also related to other factors and is directly related to the result of $\mathrm{Q}_{1}$. This result suggests that it may be a big psychological burden for young people to engage with unfamiliar people when providing services. Even people in the area are unknown to them, and it may be proof that there is little relationship with the area from day to day. This result seems reasonable 
Table 1: Prior and posterior probabilities of Bayesian network in the model with objective variable $\mathrm{Q}_{1}$

\begin{tabular}{l|cllllllll}
\hline Node & state & $\begin{array}{l}\text { Prior } \\
\text { Prob. }\end{array}$ & \multicolumn{2}{l}{$\begin{array}{l}\text { Posterior Prob. } \\
\text { hypothesis 1 }\end{array}$} & \multicolumn{2}{l}{ hypothesis 2 } & \multicolumn{2}{l}{ hypothesis 2+ } \\
\hline $\mathrm{Q}_{1}$ & 0 & 0.443 & 0.781 & 0.209 & 0.176 & 0.535 & 0.125 & 0.857 \\
$\mathrm{Q}_{1}$ & 1 & 0.557 & 0.219 & 0.791 & 0.824 & 0.465 & 0.875 & 0.143 \\
$\mathrm{P}_{1}$ & 0 & 0.744 & 0.744 & 0.744 & 0.744 & 0.744 & 0.744 & 0.744 \\
$\mathrm{P}_{1}$ & 1 & 0.256 & 0.256 & 0.256 & 0.256 & 0.256 & 0.256 & 0.256 \\
$\mathrm{P}_{2}$ & 0 & 0.744 & 0.744 & 0.744 & $\underline{0.000}$ & $\underline{1.000}$ & $\underline{0.000}$ & $\underline{1.000}$ \\
$\mathrm{P}_{2}$ & 1 & 0.256 & 0.256 & 0.256 & $\underline{1.000}$ & $\underline{0.000}$ & $\underline{1.000}$ & $\underline{0.000}$ \\
$\mathrm{P}_{3}$ & 0 & 0.678 & 0.678 & 0.678 & 0.386 & 0.778 & 0.386 & 0.778 \\
$\mathrm{P}_{3}$ & 1 & 0.322 & 0.322 & 0.322 & 0.614 & 0.222 & 0.614 & 0.222 \\
$\mathrm{P}_{4}$ & 0 & 0.659 & 0.659 & 0.659 & 0.658 & 0.721 & 0.658 & 0.721 \\
$\mathrm{P}_{4}$ & 1 & 0.341 & 0.341 & 0.341 & 0.342 & 0.279 & 0.342 & 0.279 \\
$\mathrm{~N}_{1}$ & 0 & 0.600 & $\underline{0.000}$ & $\underline{1.000}$ & 0.600 & 0.600 & 0.610 & 0.535 \\
$\mathrm{~N}_{1}$ & 1 & 0.400 & $\underline{1.000}$ & $\underline{0.000}$ & 0.400 & 0.400 & 0.390 & 0.465 \\
$\mathrm{~N}_{2}$ & 0 & 0.859 & 0.757 & 0.927 & 0.859 & 0.859 & 0.888 & 0.667 \\
$\mathrm{~N}_{2}$ & 1 & 0.141 & 0.243 & 0.073 & 0.141 & 0.141 & 0.112 & 0.333 \\
$\mathrm{~N}_{3}$ & 0 & 0.870 & 0.757 & 0.945 & 0.870 & 0.870 & 0.872 & 0.858 \\
$\mathrm{~N}_{3}$ & 1 & 0.130 & 0.243 & 0.055 & 0.130 & 0.130 & 0.128 & 0.142 \\
$\mathrm{~N}_{4}$ & 0 & 0.870 & 0.849 & 0.884 & 0.870 & 0.870 & $\underline{1.000}$ & $\underline{0.000}$ \\
$\mathrm{~N}_{4}$ & 1 & 0.130 & 0.151 & 0.116 & 0.130 & 0.130 & $\underline{0.000}$ & $\underline{1.000}$ \\
\hline
\end{tabular}

because it is similar to that of the previous study [8][9]. This result is significant in that it supports the findings of previous studies.

From Fig. 2, it was revealed that the negative reasons prepared by this paper did not affect the evaluation of $\mathrm{Q}_{2}$. Only two points, "convenience of MASS ", and "useful for saving money", are directly related to the evaluation of $\mathrm{Q}_{2}$. From Fig. 2, the negative reasons are considered not to be a big problem when using the service. We thought that young people accept MASS as a general $\mathrm{C}$ to $\mathrm{C}$ service about the major reason for this result. Or, young people might have felt their daily lives inconvenient, and they might have thought MASS effective as a solution to improve their life. In addition, Fig. 1 suggests the resistance when you serve others by using MASS, but there is no resistance to contact with other people. This trend is considered to be natural because providing service is generally concerned. The results of both Fig. 1 and Fig. 2 showed that it is relatively easy to gather users who receive services while gathering users who provide services may be difficult. Building a system that brings users who offer services is considered to be an essential issue to realize the service continuity of MASS.

When we look at the composition of the questionnaire, the content of each reason $P_{1}-P_{7}, N_{1}-N_{8}$ is mainly asking respondents' values. Based on this fact, we made some hypotheses, and these are shown below. We conducted numerical experiments using the constructed Bayesian network, verified the hypothesis, and attempted to acquire new knowledge.

\section{Hypotheses}

- Hypothesis 1: A person who is nervous (scared) to engage with unknown others will not use MASS as a service provider. 
Table 2: Posterior probabilities of Bayesian network with objective variable $\mathrm{Q}_{1}$ when giving some settings

\begin{tabular}{l|cllllll}
\hline Node & state & \multicolumn{7}{l}{ Posterior Prob. } & & & & \\
& & Setting 1 & Setting 2 & Setting 3 & Setting 4 & Setting 5 & Setting 6 \\
\hline $\mathrm{Q}_{1}$ & 0 & 0.950 & 0.098 & 0.360 & 0.411 & $\underline{0.000}$ & $\underline{1.000}$ \\
$\mathrm{Q}_{1}$ & 1 & 0.050 & 0.902 & 0.640 & 0.589 & $\underline{1.000}$ & $\underline{0.000}$ \\
$\mathrm{P}_{1}$ & 0 & 0.744 & 0.744 & 0.661 & 0.744 & 0.744 & 0.744 \\
$\mathrm{P}_{1}$ & 1 & 0.256 & 0.256 & 0.339 & 0.256 & 0.256 & 0.256 \\
$\mathrm{P}_{2}$ & 0 & $\underline{1.000}$ & $\underline{0.000}$ & 0.513 & 0.656 & 0.624 & 0.897 \\
$\mathrm{P}_{2}$ & 1 & $\underline{0.000}$ & $\underline{1.000}$ & 0.487 & 0.344 & 0.376 & 0.103 \\
$\mathrm{P}_{3}$ & 0 & 0.778 & 0.386 & $\underline{0.000}$ & 0.481 & 0.631 & 0.738 \\
$\mathrm{P}_{3}$ & 1 & 0.222 & 0.614 & $\underline{1.000}$ & 0.519 & 0.369 & 0.262 \\
$\mathrm{P}_{4}$ & 0 & 0.721 & 0.658 & 0.531 & $\underline{0.000}$ & 0.641 & 0.693 \\
$\mathrm{P}_{4}$ & 1 & 0.279 & 0.342 & 0.469 & $\underline{1.000}$ & 0.359 & 0.307 \\
$\mathrm{~N}_{1}$ & 0 & $\underline{0.000}$ & $\underline{1.000}$ & 0.600 & 0.600 & 0.847 & 0.295 \\
$\mathrm{~N}_{1}$ & 1 & $\underline{1.000}$ & $\underline{0.000}$ & 0.400 & 0.400 & 0.153 & 0.705 \\
$\mathrm{~N}_{2}$ & 0 & 0.757 & 0.927 & 0.859 & 0.859 & 0.913 & 0.782 \\
$\mathrm{~N}_{2}$ & 1 & 0.243 & 0.073 & 0.141 & 0.141 & 0.087 & 0.218 \\
$\mathrm{~N}_{3}$ & 0 & 0.757 & 0.945 & 0.870 & 0.870 & 0.917 & 0.812 \\
$\mathrm{~N}_{3}$ & 1 & 0.243 & 0.055 & 0.130 & 0.130 & 0.083 & 0.188 \\
$\mathrm{~N}_{4}$ & 0 & 0.849 & 0.884 & 0.870 & 0.870 & 0.949 & 0.761 \\
$\mathrm{~N}_{4}$ & 1 & 0.151 & 0.116 & 0.130 & 0.130 & 0.051 & 0.239 \\
\hline
\end{tabular}

- Hypothesis 2: A person who wants to get rewards will want to use MASS as a service provider.

- Hypothesis 3: A person who feels MASS useful will want to use MASS as a client.

- Hypothesis 4: A person who wants to join the interaction with local people will use MASS as a client.

- Hypothesis 5: A person who wants to want to reduce waste/to save money will use MASS as a client.

We set the evidence for the item corresponding to each hypothesis shown above. We also set up contrapositive evidence to increase the accuracy of explanation and perform numerical experiments. In Hypothesis 1, we investigate the changes of all probability by setting the evidence for two states $\mathrm{N}_{1}=0$ and $\mathrm{N}_{1}=1$. The evidence for Hypothesis 2 is $\mathrm{P}_{2}$, the evidence for Hypothesis 3 is $\mathrm{P}_{7}$, the evidence for Hypothesis 4 is $\mathrm{P}_{5}$, and the evidence for Hypothesis 5 is $\mathrm{P}_{6}$.

Table 1 and Table 3 summarize the prior probabilities and posterior probabilities about above mentioned hypotheses. In order to provide additional considerations, we performed numerical experiments with some evidences, and the results are shown in Table 2 and Table 4 respectively. The experimental result of setting evidence shows numerical values supporting Hypothesis 1, Hypothesis 3, and these contrapositive hypotheses. Concerning Hypothesis 2, we could have obtained its contrapositive hypothesis by adding evidence to $\mathrm{N}_{4}$ (Hypothesis 2+), which can derive the opposite result. In Hypothesis 2, the probability of $\mathrm{Q}_{1}=0$ when $\mathrm{P}_{2}=0$ is much lower and asymmetric than the probability of $\mathrm{Q}_{1}=1$ when $\mathrm{P}_{2}=1$. This tendency suggests that the negative factors determining $\mathrm{Q}_{1}=0$ are complex. Then, we added Hypothesis 2+ to investigate negative factors in more detail, which added $\mathrm{N}_{4}$ as evidence to Hypothesis 2. In Hypothesis 2+, the probabilities when the evidence 
Table 3: Prior and posterior probabilities of Bayesian network in the model with objective variable $\mathrm{Q}_{2}$

\begin{tabular}{l|cccccccc}
\hline Node & state & $\begin{array}{l}\text { Prior } \\
\text { Prob. }\end{array}$ & \multicolumn{2}{l}{ Posterior Prob. } \\
& & hypothesis 3 & \multicolumn{2}{l}{ hypothesis 4 } & \multicolumn{2}{l}{ hypothesis 5 } \\
\hline $\mathrm{Q}_{2}$ & 0 & 0.482 & 0.072 & 0.817 & 0.171 & 0.544 & 0.170 & 0.526 \\
$\mathrm{Q}_{2}$ & 1 & 0.518 & 0.928 & 0.183 & 0.829 & 0.456 & 0.830 & 0.474 \\
$\mathrm{P}_{5}$ & 0 & 0.878 & 0.807 & 0.930 & $\underline{0.000}$ & $\underline{1.000}$ & 0.928 & 0.869 \\
$\mathrm{P}_{5}$ & 1 & 0.122 & 0.193 & 0.070 & $\underline{1.000}$ & $\underline{0.000}$ & 0.072 & 0.131 \\
$\mathrm{P}_{6}$ & 0 & 0.858 & 0.863 & 0.855 & 0.917 & 0.850 & $\underline{0.000}$ & $\underline{1.000}$ \\
$\mathrm{P}_{6}$ & 1 & 0.142 & 0.137 & 0.145 & 0.083 & 0.150 & $\underline{1.000}$ & $\underline{0.000}$ \\
$\mathrm{P}_{7}$ & 0 & 0.578 & $\underline{0.000}$ & $\underline{1.000}$ & 0.333 & 0.613 & 0.592 & 0.576 \\
$\mathrm{P}_{7}$ & 1 & 0.422 & $\underline{1.000}$ & $\underline{\underline{0.000}}$ & 0.667 & 0.388 & 0.408 & 0.424 \\
\hline
\end{tabular}

Table 4: Posterior probabilities of Bayesian network with objective variable $\mathrm{Q}_{2}$ when giving some settings

\begin{tabular}{l|lllllll}
\hline Node & state & $\begin{array}{l}\text { Posterior Prob. } \\
\text { Setting 7 }\end{array}$ & Setting 8 & Setting 9 & Setting 10 & Setting 11 & Setting 12 \\
\hline $\mathrm{Q}_{2}$ & 0 & 0.500 & 0.615 & 0.500 & 0.978 & $\underline{0.000}$ & $\underline{1.000}$ \\
$\mathrm{Q}_{2}$ & 1 & 0.500 & 0.385 & 0.500 & 0.022 & $\underline{1.000}$ & $\underline{0.000}$ \\
$\mathrm{P}_{5}$ & 0 & $\underline{0.000}$ & $\underline{1.000}$ & $\underline{0.000}$ & $\underline{1.000}$ & 0.768 & 0.966 \\
$\mathrm{P}_{5}$ & 1 & $\underline{1.000}$ & $\underline{\underline{0.000}}$ & $\underline{\underline{1.000}}$ & $\underline{0.000}$ & 0.232 & 0.034 \\
$\mathrm{P}_{6}$ & 0 & $\underline{\underline{0.000}}$ & $\underline{1.000}$ & $\underline{0.000}$ & $\underline{1.000}$ & 0.800 & 0.950 \\
$\mathrm{P}_{6}$ & 1 & $\underline{1.000}$ & $\underline{0.000}$ & $\underline{1.000}$ & $\underline{0.000}$ & 0.200 & 0.050 \\
$\mathrm{P}_{7}$ & 0 & 0.333 & 0.613 & $\underline{\underline{0.000}}$ & $\underline{\underline{1.000}}$ & 0.240 & 0.952 \\
$\mathrm{P}_{7}$ & 1 & 0.667 & 0.388 & $\underline{\underline{1.000}}$ & $\underline{\underline{0.000}}$ & 0.760 & 0.048 \\
\hline
\end{tabular}

$\mathrm{P}_{2}=1$ and $\mathrm{N}_{4}=0$ was set, which is usual condition, and the probabilities when $\mathrm{P}_{2}=0$ and $\mathrm{N}_{4}=1$ were almost symmetric. From this result, we can understand that $\mathrm{N}_{4}$ is a major factor as a negative reason for $\mathrm{Q}_{1}$. In Hypothesis 3, as in Hypothesis 2, the probability of $\mathrm{Q}_{2}=0$ when $\mathrm{P}_{7}=0$ is slightly lower than the probability of $\mathrm{Q}_{2}=1$ when $\mathrm{P}_{7}=1$. Therefore, in the case of Hypothesis 3, it is suggested that the factor determining $\mathrm{Q} 2=0$ is not as complicated as that of Hypothesis 2 but is complicated. In Hypotheses 4 and 5, as in Hypothesis 2, it is suggested that the factors of the negative evaluation are complex. At this point, we can clarify the negative factors in the same way as the processing performed in Hypothesis 2.

We gave various patterns of evidence and found some notable findings. Table 2 and Table 4 show the 12 types of evidence settings described as Setting1 to Setting12. The evidence of Setting 2 and Setting 1 are from the knowledge that these settings have decided $\mathrm{Q}_{1}$ with a high probability.

From these results, the strategy to encourage the use of people corresponding to the evidence of Setting 2, who wants a reward and is willing to interact with others, would be particularly important for the spread of MASS. While we found that some factors were giving great influence, like Setting 2, as shown in Settings 3 and 4, it became clear that the motivation for participating in the community did not significantly affect the result of $Q_{1}$. Youths indeed have low interest in the community. As one of the motivations of MASS, we 
have been examining to introduce MASS as a means to raise local interest. In this regard, Settings 3 and 4 reconfirmed the significance of promoting MASS. Settings 5 and 6 are for confirming the magnitude of the effect of the explanatory variables. Setting 6 shows the strength of $r m N_{1}$. From Setting 6, we can also confirm that $r m N_{1}$ is a particularly important factor.

Next, we show the changes in probability for $\mathrm{Q}_{2}$ in Table 4 . As shown in Fig. $2, \mathrm{~N}_{5}-\mathrm{N}_{8}$ does not affect the objective variable, so only $\mathrm{P}_{5}-\mathrm{P}_{7}$ for which evidence is set it's shown. As previously mentioned, we could have found the strength of the effect of $\mathrm{P}_{7}$, which is the correctness of Hypothesis 3. In order to investigate the impact of factors related to $\mathrm{Q}_{2}$, we experimented with Settings 7 and 8. We checked the posterior probability of these settings and found that the two factors did not significantly affect MASS. Since there are only three factors directly related to $\mathrm{Q}_{2}$, we can imagine the strength of the factor of $\mathrm{P}_{7}$ from these results. However, Setting9 did not show that, despite the opposite, Setting 10 showed the expected results. Experiments in Settings 11 and 12 were to clarify the contradictions shown in Settings 9 and 10. The results showed that $\mathrm{P}_{5}-\mathrm{P}_{7}$ were not independent. Specifically, to get a positive opinion in $\mathrm{Q}_{2}$, we found that $\mathrm{P}_{7}$ must be 1 and $\mathrm{P}_{5}$ and $\mathrm{P}_{6}$ must be negative. The finding, the motivation of youth using MASS is only the convenience rather than interacting with others and reducing waste, is surprising. This paper would have shown significant results in that appealing only to convenience was important for user acquisition. However, the most important users of this study are the vulnerable road users. Clarifying the differences in awareness between youth and the elderly will be absolutely necessary for the future. This study would have enough contribution because it has clarified the future direction of MASS, as shown above.

\section{Conclusion}

This paper analyzed young people's awareness of MASS with Bayesian network to understand their mechanism of the evaluation for MASS. From the results of examining the structure of Bayesian network, we confirmed the findings of the previous research and further clarified the relationship between the factors that we could not find so far. Specifically, when providing MASS services, we confirmed the possibility that the setting of remuneration is important, and the interaction with others becomes a psychological burden because people in the area are unknown to many young people. When getting services with MASS, although young people do not need MASS to interact with and contribute to the region, they may have accepted MASS as a general $\mathrm{C}$ to $\mathrm{C}$ service. It may be relatively easy to acquire users who receive services, while it may be difficult to gather users who provide their resources. Therefore, we found that the strategy to gather users who offer services is a challenge for the spread of MASS.

Next, we conducted numerical experiments using the constructed Bayesian network, obtained results that support several hypotheses, and revealed factors that have a strong influence on the use of MASS. The experimental result showed the changes of probability supporting all hypotheses prepared by this paper. People who want rewards and are not resistant to interacting with others can expect to participate in MASS with high probability as users who provide services. Therefore, the strategy to encourage such people would be particularly important for the spread of MASS. When using the MASS service, we found a possibility that the psychological resistance to engaging with others has a strong influence on the use of MASS. At the same time, it became clear that the motivation for participating 
in the community was not a significantly important factor to promote a user who provides his/her resource. We found that convenience is a strong factor that transcends the influence of various negative factors when using the service of MASS.

This paper clarified four modules that affect the successful implementation; skill provider's mind, demander's mind, needs, and emotion to reactivate the local community by overcoming the fears of connecting with unknown people and usability of MASS. We will design some research strategy as one of the important future works in integrating these four aspects.

As future work, we will also construct Bayesian networks with the following three questions as objective functions and the reasons used by this paper as explanatory variables.

- Do you think that local residents will use MASS? Give your opinion from an objective standpoint.

- Do you think that local residents will use MASS to share resources? Give your opinion from an objective standpoint.

- Do you think a service that supports local people's mutual aid without relying on public services ideal?

\section{Acknowledgment}

This work was partly supported by Japan Society for the Promotion of Science, Grant-inAid for Scientific Research (C), No.18K02214 and FIVE, Non-Profit Organization.

\section{References}

[1] Banai-Kashanai, A.R., Toward a Synthetic Measure of Good Settlement Form" Environment and Planning B, 15, pp.399-412 (1988).

[2] N. Ohigashi and S. Matsumoto, Research on the Possibility of Riding-together Traffic in Residential Estates, Bulletin of Hiroshima Institute of Technology, Research, Vol.49, pp.19-22 (2015), In Japanese.

[3] S. Matsumoto and N. Ohigashi, Examining an Effective Way to Support Vulnerable Road Users in Itsukaichi District, Hiroshima City, Information Engineering Express, Special issue on Business Management of Technology, Vol.2, No.3, pp.43-52 (2016).

[4] D. Demailly, A. Novel, The sharing economy: make it sustainable. Studies, (03/14), 30 (2014).

[5] J. Hamari, M. Sjoklint, A. Ukkonen, The sharing economy: Why people participate in collaborative consumption. Journal of the Association for Information Science and Technology, (2015).

[6] S. Matsumoto, N. Ohigashi, T. Hasuike, Developing a Transportation Support System for Vulnerable Road Users in Local Community, Proc. of 2016 5th IIAI International Congress on Advanced Applied Informatics, pp.797-800 (2016).

[7] S. Matsumoto, N. Ohigashi and T. Hasuike, Design and Development of a Web Service to Support Daily Life of Vulnerable Road Users in Suburban Residential Estates in 
Hiroshima City, Proc. of 2017 6th IIAI International Congress on Advanced Applied Informatics, In USB (2017).

[8] S. Matsumoto, N. Ohigashi, T. Hasuike, Livelihood Assistance System for Vulnerable Road Users in Suburban Residential Areas based on Mutual Assistance, International Journal of Service and Knowledge Management, ISSN: 2189-9231, Vol 1, No 2, pp.1331 (2017).

[9] S. Matsumoto, N. Ohigashi, Attitude Survey of Young People to Examine the Usefulness of a Skill Sharing Web Service for Regional Vulnerable Road Users, Proceeding of 7th International Congress on Advanced Applied Informatics, pp.724-729 (2018).

[10] T. Dillahunt, A. Malone, The promise of the sharing economy among disadvantaged communities. In Proceedings of the 33rd Annual ACM Conference on Human Factors in Computing Systems, pp.2285-2294. ACM (2015).

[11] D. Rauch, D. Schleicher, Like uber, but for local government law: The future of local regulation of the sharing economy. Ohio St. LJ, 76, 901 (2015).

[12] D. Wosskow, Unlocking the sharing economy: An independent review (2014).

[13] S. Denning, An economy of access is opening for business: five strategies for success. Strategy \& Leadership, 42(4), pp.14-21 (2014).

[14] J. Pearl, Bayesian Networks: a Model of Self-Activated Memory for Evidential Reasoning, Proc of Cognitive Science Society, pp.329-334 (1985).

[15] F. Jensen, An Introduction to Bayesian networks, University College London Press (1996).

[16] E. Castillo, J. Gutierrez, and A.Hadi, Expert systems and probabilistic network models, Springer-Verlag (1997).

[17] R. Cowell, A. Dawid, S. Lauritzen, and D. Spiegelhalter, Probabilistic Networks and Expert Systems, Springer-Verlag (1999).

[18] Y. Motomura, Bayesian Network Softwares, Journal of the Japanese Society for Artificial Intelligence, Vol.17, No.5, pp.559-565 (2002).

[19] S. Matsumoto, N. Ohigashi, Analyzing Young People's Awareness for Mutual Assistance Support System with Bayesian Network, Proc. of 2019 8th International Congress on Advanced Applied Informatics (IIAI-AAI), pp.762-767 (2019).

[20] S. Russell, P. Norvig : “Artificial Intelligence A Modern Approach ”, Prentice Hall Series in Artificial Intelligence (1995)

[21] R. E. Neapolitan : ” Learning Bayesian Networks", Artificial Intelligence, Prentice Hall (2004) 